\title{
Wer bin ich - und was kann ich dagegen tun? Fiktionale Wahrheit und mimetisch unzuverlässiges Erzählen in Max Frischs Stiller (1954)
}

\section{Victor Lindblom}

Zusammenfassung Am Beispiel des Romans Stiller von Max Frisch wird im Beitrag die These plausibel gemacht, dass mimetisch unzuverlässiges Erzählen zu einer Aufspaltung einer fiktiven Welt in mehrere - potenziell unterschiedlich stark autorisierte - fiktive Welten führt. Dieser Auffassung zufolge wird durch das unzuverlässige Erzählen des Protagonisten der Roman in eine Stiller-Fiktion und in eine White-Fiktion unterteilt. Beide fiktive Welten bestehen demnach aus fiktionalen Wahrheiten, jedoch wird die Stiller-Fiktion durch den Autor stärker autorisiert als die White-Fiktion. Diese Erzählkonzeption verlangt nach einer Interpretation des Romans aus zwei Perspektiven, die im Identitätsproblem ihren gemeinsamen Kern haben: In derselben Weise wie Stiller nicht Whites Identität annehmen kann, kann White nicht Stillers Identität annehmen.

„Ich bin nicht Stiller!“ (Frisch 1976, S. 361) - Ich werde mich hier vor allem mit diesem ersten Satz aus Max Frischs Roman Stiller auseinanderersetzen. Dabei interessiert mich in erster Linie die Beziehung zwischen fiktionaler Wahrheit und mimetisch unzuverlässigem Erzählen. Es geht somit um die Beziehung zwischen (a) dem Bestehen von Sachverhalten in fiktiven Welten und (b) einem Erzählverhalten, das am Bestehen von Sachverhalten in fiktiven Welten zweifeln lässt. Zunächst ein Umriss der These.

\footnotetext{
V. Lindblom $(\bowtie)$

Departement für Germanistik,

Universität Fribourg,

Freiburg, Schweiz

E-Mail: victor.lindblom@unifr.ch
} 


\section{These}

Der erste Eintrag des Stiller-Protagonisten in sein Tagebuch ist die Behauptung, dass er nicht Stiller sei. Vor diesem Satz hat der Leser jedoch die Information erhalten, dass es sich beim Folgenden um „Stillers Aufzeichnungen im Gefängnis“ (Frisch 1976, S. 361) handeln soll.

Der Leser wird, so scheint es zumindest bei der ersten Lektüre, mit einem Widerspruch konfrontiert. Proposition $s$ lautet, dass es sich beim Protagonisten um Stiller handelt. Proposition $w$ lautet, dass es sich beim Protagonisten um White handelt. Keine dieser Propositionen sollte nun aber, wie ich vorschlagen möchte, als fiktional falsch klassifiziert werden. Weder $s$ noch $w$ ist in demselben Sinne fiktional falsch wie zum Beispiel die Propositionen, dass der Protagonist Analphabet oder Chirurg oder Österreicher (usw.) ist, fiktional falsch sind.

Es sollten vielmehr beide Propositionen als fiktional wahr klassifiziert werden, obwohl sie sich widersprechen. Proposition $s$ ist fiktional wahr in der fiktiven Welt der Stiller-Fiktion. Proposition $w$ ist fiktional wahr in der fiktiven Welt der White-Fiktion. Dabei sind die Propositionen $s$ und $w$ auf unterschiedliche Weisen fiktional wahr. Proposition $s$ drückt als Teil der Stiller-Fiktion eine autorisierte fiktionale Wahrheit aus. Proposition $w$ drückt als Teil der White-Fiktion eine unautorisierte fiktionale Wahrheit aus.

Mimetisch unzuverlässiges Erzählen führt, so die zu erläuternde These, zu einer Aufspaltung von einer fiktiven Welt in mindestens zwei - potenziell unterschiedlich stark autorisierte - fiktive Welten. Im Folgenden werde ich zunächst darauf eingehen, was ich unter fiktionaler Wahrheit und fiktiven Welten verstehe. Dabei handelt es sich um die fiktionstheoretische Basis der anschließenden Überlegungen zum unzuverlässigen Erzählen. Zuletzt werde ich darauf aufbauend eine Interpretation (bzw. zwei Interpretationen) von Stiller skizzieren.

\section{Fiktionale Wahrheit und fiktive Welten}

Was ist eine fiktionale Wahrheit? Ein Satz ,p“ ist wahr, wenn $p$ in der Welt der Fall ist. Ein Satz ,p“ ist fiktional wahr, wenn $p$ in einer bestimmten fiktiven Welt der Fall ist. Wahr zu sein und fiktional wahr zu sein sind mögliche Eigenschaften von Sätzen. Soweit die übliche Terminologie, an die ich mich halte. ${ }^{1}$ Entscheidend ist nun aber die Frage: Wie kommt es dazu, dass etwas in einer fiktiven Welt der Fall ist? Wann ist eine Proposition fiktional wahr?

\footnotetext{
${ }^{1}$ Ich problematisiere also den Wahrheitsbegriff hier nicht, sondern benutze das Wort wahr, wie es im Alltag und in der Wissenschaft in aller Regel (auf der Basis einer Korrespondenztheorie) verwendet wird. Vgl. jedoch zur Nicht-Zerlegbarkeit des Wahrheitsbegriffs und den gängigen Wahrheitstheorien als Wahrheitsfeststellungstheorien etwa Gabriel (2015, S. 37-39).
} 
An dieser Stelle kann ich verschiedene Theorien fiktionaler Wahrheit nicht gebührend vergleichen. ${ }^{2}$ Eine weit verbreitete Auffassung, der ich mich anschließe, ist aber: In fiktiven Welten ist, grob gesagt, das der Fall, was man sich auf der Basis eines fiktionalen Textes (oder einer fiktionalen Textpassage) vorstellen soll.

Diese Grundidee bringt etwa Kendall Walton auf den Punkt: „A fictional truth consists in there being a prescription or mandate [...] to imagine something " (Walton 1990, S. 39). Eine fiktionale Wahrheit (bzw. fiktive Tatsache ${ }^{3}$ ) ist demnach das Ergebnis einer Vorschrift, Anordnung oder Aufforderung sich etwas vorzustellen. Wird aktiv eine Vorstellung verschrieben, entsteht eine fiktionale Wahrheit. Es stellt sich insofern die Folgefrage: Wer oder was verschreibt solche Vorstellungen? Wie werden fiktionale Wahrheiten generiert?

Walton etwa ist der Auffassung, dass fiktionale Wahrheiten nicht nach einem einzelnen, benennbaren Prinzip generiert werden. Es müsse vielmehr von Fall zu Fall entschieden werden, welche fiktionalen Wahrheiten auf welche Weise generiert werden. ${ }^{4}$ Andere sind der Auffassung, dass man durchaus benennen oder zumindest gewinnbringend stipulieren kann, woher die für die Generierung fiktionaler Wahrheiten entscheidende Aufforderung zur Imagination kommt.

Eine naheliegende und plausible Antwort ist: vom Verfasser des Textes. Die Theorie fiktionaler Wahrheit, an die ich mich hier halten werde, ist Kathleen Stocks Variante eines starken Intentionalismus: ${ }^{5}$ „The basic idea [...] is that the fictional content of a particular text is equivalent to exactly what the author of the text intended the reader to imagine" (Stock 2017, S. 1). Im Detail:

An author $A u$ 's utterance $x$ (or set of utterances $S$ ) has fictional content that $p$, if and only if: $A u$ utters $x$ (or $S$ ) intending that i) $x$ (or $S$ ) should cause F-imagining that $p$ in her intended readership $R$; ii) $R$ should recognize this intention; and iii) $R$ 's recognition of this intention should function as part of $R$ 's reason to F-imagine that $p$. (Stock 2017, S. 15)

Eine fiktionale Wahrheit $p$ wird demnach genau dann generiert, wenn der Autor mit der reflexiven Intention eine Äußerung $x$ (in aller Regel: ,„p“) tätigt, dass der

\footnotetext{
${ }^{2}$ Für einen Überblick vgl. Köppe (2014).

${ }^{3}$ Die im Deutschen allesamt gebräuchlichen Ausdrücke fiktionale Wahrheit, fiktive Tatsache und fiktiver Fakt behandle ich im Folgenden im Sinne des Englischen fictional truth vereinfachend als synonym.

${ }^{4}$ „For purposes of divining fictional truths there is no substitute for a good nose: a combination of imagination and common sense, leavened within limits by charity and informed by familiarity with the medium, genre, and representational tradition to which the work in question belongs as well as by knowledge of the outside world - all of this combined, of course, with sensitivity to the most subtle features of the work itself" (Walton 1990, S. 184). Allgemein zur Ermittlung fiktionaler Wahrheiten in fiktiven Welten vgl. auch Köppe (2008, S. 65-89); Zipfel (2001, S. 84-90; 2011).

${ }^{5}$ Die These, die ich zur Diskussion stellen möchte, steht und fällt aber nicht mit dieser Auffassung: An dieser Stelle könnte vermutlich auch ein hypothetischer Intentionalismus oder Textintentionalismus vertreten werden, ohne dass sich dadurch etwas an der grundlegenden Idee ändert.
} 
Leser sich vorstellen soll, dass $p .{ }^{6}$ Auf dieser Basis definiert Stock eine Fiktion: ,[A] fiction is, necessarily and sufficiently, a collection of propositions reflexively intended by their author to be conjoined in imagination" (Stock 2017, S. 158).

Unter einer fiktiven Welt verstehe ich dasselbe wie Stock unter einer Fiktion: Eine fiktive Welt ist eine durch einen Autor generierte Menge an in der Vorstellung zu verknüpfenden fiktionalen Wahrheiten. Eine bestimmte fiktive Welt besteht aus einer bestimmten Menge an fiktionalen Wahrheiten. Wie die Welt als die Summe ihrer Tatsachen verstanden werden kann, ${ }^{7}$ kann eine fiktive Welt als die Summe ihrer fiktiven Tatsachen verstanden werden. ${ }^{8}$

\section{Was ist der Fall?}

Wenn es in Stiller zunächst heißt, dass es sich beim Folgenden um „Stillers Aufzeichnungen im Gefängnis“ handelt, der erste Satz dieser Aufzeichnungen aber „Ich bin nicht Stiller!“ (Frisch 1976, S. 361) lautet, stehen wir als Leser zu diesem Zeitpunkt der Lektüre vor zwei sich widersprechenden Vorstellungsaufforderungen.

Zuerst verschreibt uns Frisch die Vorstellung, dass es sich bei den kommenden sieben Heften um die Aufzeichnungen eines Mannes namens Stiller handelt. Im zweiten Teil des Romans, dem "Nachwort des Staatsanwalts“ (Frisch 1976, S. 730), erfahren wir zudem, dass es der Staatsanwalt Rolf ist, der diese Hefte unter dem Titel Stillers Aufzeichnungen im Gefängnis herausgegeben und kommentiert hat. Die Stiller-Fiktion fordert uns also unter anderem zu den Vorstellungen auf, dass es sich beim Protagonisten um Ludwig Anatol Stiller handelt, der James Larkin White sein möchte, aber von seinem Umfeld und der Justiz dazu gezwungen wird, wieder Stiller zu sein.

Mit dem ersten Satz aus Stillers Aufzeichnungen verschreibt uns Frisch aber auch die Vorstellung, dass der Protagonist behauptet, nicht Stiller zu sein. Diese

\footnotetext{
${ }^{6} \mathrm{Zu}$ reflexiven Intentionen vgl. Stock (2017, S. 16-19, 149) sowie grundlegend Grice (1957, S. 383). Die Vorstellungen, zu denen der Autor auffordert, sind nach Stock durch drei Merkmale bestimmt: Sie sind 1) propositional (ihr Gehalt ist die im Satz direkt oder durch den Satz indirekt ausgedrückte Proposition), 2) quasi-faktual (der Leser stellt sich vor, dass die vom Satz ausgedrückte Proposition wahr ist) und 3) potentiell konjunktiv (der Leser verknüpft die propositionalen und quasi-faktualen Vorstellungen, falls weitere hinzukommen, mit diesen). Dieses fiktionsspezifische Vorstellen fasst Stock als F-imagining zusammen (vgl. Stock 2017, S. 145148). Für eine Rekonstruktion und Anwendung von Stocks Fiktionstheorie vgl. auch Lindblom (2020).

${ }^{7}$ Nach Wittgensteins „Die Welt ist alles, was der Fall ist“ (Tractatus 1).

${ }^{8}$ Vgl. zu fiktiven Welten als Mengen von Propositionen auch Walton (1990, S. 66-67); Köppe (2014, S. 205).
} 
Behauptung wiederholt der Protagonist immer wieder und erzählt nicht als Stiller, sondern als White von sich und von Stiller. Die White-Fiktion fordert uns also unter anderem $\mathrm{zu}$ den Vorstellungen auf, dass es sich beim Protagonisten um James Larkin White handelt, der aber von seinem Umfeld und der Justiz dazu gezwungen wird, Stiller zu werden.

Was ist der Fall?

\section{Mimetisch unzuverlässiges Erzählen}

Damit kommt die Frage der Zuverlässigkeit ins Spiel. Zunächst zur Terminologie: „[D]er Terminus ,mimetische Unzuverlässigkeit“ soll für die [...] Erzähler stehen, die ihren jeweiligen Gegenstand nicht angemessen darstellen“ (Kindt 2008, S. 48). Dieses grundlegende Prinzip des unzuverlässigen Erzählens scheint mir in folgender Definition von Tilmann Köppe und Tom Kindt am klarsten auf den Punkt gebracht:

The narration expressed by a literary work $\mathrm{W}$ is mimetically unreliable if, and only if, $\mathrm{W}$ authorizes (as part of the composition strategy $\mathrm{w}_{\mathrm{w}}$ ) readers to imagine that the narrator does not provide completely accurate information or all relevant information. (Köppe und Kindt 2011, S. 85) ${ }^{9}$

Ein Erzähler ist demnach genau dann mimetisch unzuverlässig, wenn der Leser sich vorstellen soll, dass i) dessen Sätze nicht fiktional wahr oder in relevanter Weise unvollständig sind und ii) dies im Kontext der Kompositionsstrategie des Werks eine bestimmte Funktion erfüllt. ${ }^{10}$

Wenn ein Erzähler unzuverlässig ist, handelt es sich also bei diesem Sachverhalt um eine fiktive Tatsache: Der Autor fordert den Leser auf, sich die fiktiven

\footnotetext{
${ }^{9}$ Für das hier verfolgte Ziel ist eine adäquate Bestimmung dieses allgemeinen Prinzips des mimetisch unzuverlässigen Erzählens ausreichend. Köppe und Kindt unterscheiden später (im Anschluss an Stühring 2011) zudem zwischen offen und täuschend unzuverlässigem Erzählen je nachdem, ob der Leser über die fiktionale Wahrheit, dass ein Erzähler unzuverlässig ist, vorübergehend im Unklaren gelassen wird (täuschend) oder nicht (offen) (vgl. Köppe und Kindt 2014, S. 236-256). Für eine fundierte Kritik dieser Unterscheidung vgl. Lang [im Erscheinen]. Für eine umfassendere Typologie unzuverlässigen Erzählens vgl. Jacke (2018).

${ }^{10}$ Akkurat verstehe ich als synonym mit fiktional wahr: Wenn die Äußerungen eines Erzählers akkurat sind, sind sie fiktional wahr und bringen fiktive Tatsachen zum Ausdruck. Zur Notwendigkeit des Verweises auf die allgemeine Kompositionsstrategie des Werks in der Definition vgl. Kindt: ,[Es] lässt sich von einem ,mimetisch unzuverlässigen Erzähler' sinnvollerweise nur dann sprechen, wenn sich die Eigenschaften seiner Ausführungen, die eine Infragestellung seiner Vertrauenswürdigkeit nach sich ziehen, nicht durch die Entstehung, sondern durch die Idee des Werks zu motivieren ist. Die Zuschreibung des Konzepts setzt mit anderen Worten eine Interpretationshypothese voraus, die den festgestellten Seltsamkeiten einer Erzähläußerung eine werkbezogene Funktion zuweist" (Kindt 2008, S. 60).
} 
Erzählerfiguren (wenn er solche erfunden hat ${ }^{11}$ ) als mehr oder weniger zuverlässig vorzustellen und generiert so eine fiktional wahre Proposition. Diese fiktionale Wahrheit - man könnte sie insofern als höherstufig bezeichnen - kann wiederum den fiktionalen Wahrheitswert all jener Propositionen beeinflussen, die vom Bericht des unzuverlässigen Erzählers abhängen. Erkennen kann der Leser die mimetische Unzuverlässigkeit eines Erzählers daran, dass der Autor ihn gegen Grice'sche Konversationsmaximen - Quantität, Qualität, Relation, Modalität verstoßen lässt. ${ }^{12}$

\section{Wer ist zuverlässiger?}

Wie zeichnet also Frisch seine fiktiven Erzählerfiguren aus?

Einerseits verschreibt er dem Leser die Vorstellung, dass der Staatsanwalt als Erzähler unzuverlässig ist. So schreibt etwa der Protagonist in seinen Heften über den Staatsanwalt:

Es stimmt also nicht ganz, was mein Staatsanwalt zuvor behauptet hat; er wußte, wenn auch nicht durch Sibylle, den Namen ihres Freundes, bevor Stiller verschollen war. Ich erwähne das nur als Beispiel, daß selbst ein Staatsanwalt in seinen durchaus freiwilligen Berichten nicht ganz so widerspruchslos redet, wie sie es von unsereinem in den Verhören erwarten! (Frisch 1976, S. 579)

Andererseits verschreibt Frisch dem Leser die Vorstellung, dass der Protagonist als Erzähler unzuverlässig ist. So schreibt etwa der Staatsanwalt in seinem Nachwort über den Protagonisten:

Zu jener Zeit kannte ich die vorliegenden Aufzeichnungen noch nicht, wußte allerdings, daß Stiller in der Untersuchungshaft etwas wie ein Tagebuch geführt hatte. Es ist nicht der Sinn dieses Nachwortes, daß ich mich in zahllosen Berichtigungen ergehe. Die Mutwilligkeit seiner Aufzeichnungen, seine bewußte Subjektivität, wobei Stiller auch vor gelegentlichen Fälschungen nicht zurückschreckt, scheinen mir offenkundig genug zu sein; als Rapport über ein subjektives Erlebnis mögen sie redlich sein. (Frisch 1976, S. 749)

Beide fiktiven Erzähler behaupten implizit, wenn sie von Widersprüchlichkeiten und Fälschungen sprechen, der jeweils andere verletze die Grice'sche Maxime der Qualität. Weil der Leser sich vorstellen soll, dass ebendies der Fall ist, wurden, so scheint es wiederum zumindest bei der ersten Lektüre, fiktionale Wahrheiten generiert.

Gleichwohl hat der Leser gute Gründe, um den Staatsanwalt für insgesamt zuverlässiger zu halten als den Protagonisten. Niemand außer Knobel - und

\footnotetext{
${ }^{11}$ Für erzählerloses unzuverlässiges Erzählen passen Köppe und Kindt die Definition wiefolgt an: „The narration expressed by a literary work $\mathrm{W}$ is mimetically unreliable if, and only if, $\mathrm{W}$ does not authorize imagining that there is a narrator; instead $\mathrm{W}$ seemingly, or prima facie, authorizes imagining states of affairs that are not completely accurate" (Köppe und Kindt 2011, S. 90).

${ }^{12}$ Vgl. dazu grundlegend Grice 1975 sowie die Operationalisierung von Kindt (2008, S. 53-67).
} 
auch er nur zu Beginn - glaubt, dass dieser White sei. Der Roman ist zwar so konzipiert, dass der Leser sich durchaus wiederholt fragen kann, ob es sich beim Protagonisten um Stiller handelt. Letztlich wäre aber die Annahme - wenn man sich entscheiden müsste - besser begründet, dass es sich um Stiller handelt, der am Versuch scheitert, die White-Identität anzunehmen. Die fiktive Welt der StillerFiktion ist insofern - wie ich sie nennen möchte und noch ausführen werde - die von Frisch autorisierte.

\section{Und White?}

Wenn die Stiller-Fiktion die autorisierte ist: Was geschieht mit der White-Fiktion? Besteht diese aus fiktional falschen Propositionen? Ist es schlicht nicht der Fall, dass es sich beim Protagonisten um White handelt, der gezwungen wird, Stiller zu sein? Dies scheint zumindest die aus der Standardposition folgende Antwort zu sein, die ich infrage stellen möchte.

So würden etwa Köppe und Kindt die Propositionen der White-Fiktion wohl nicht als fiktional wahr bezeichnen. Weil nach ihrer Verwendung des Begriffs der Autorisierung nur eine tatsächlich autorisierte Vorstellung eine fiktionale Wahrheit generiert, generiert eine nur scheinbar autorisierte Vorstellung keine fiktionale Wahrheit, sondern eine fiktionale Falschheit:

Since authorized imaginings [...] constitute fictional facts, one cannot say that a text authorizes $p$, but $p$ is not a fact in the fictional world (and hence imagining that $p$ is not accurate). We might say, though, that in unreliable narration there is a difference between seemingly, or prima facie, authorized imaginings and actually authorized ones. It is only in the course of our reading and, eventually, on the basis of an elaborate interpretation that we come to realize that not all seemingly authorized imaginings are actually authorized. (Köppe und Kindt 2011, S. 89)

Auch Stock entfernt Propositionen wie jene, die nach dem präsentierten Vorschlag die White-Fiktion ausmachen, aus der Menge an fiktionalen Wahrheiten, indem sie ihre ursprüngliche Definition fiktionaler Wahrheit anpasst:

An author $A u$ 's utterance $x$ (or set of utterances $S$ ) in fiction $F$ has fictional content that $p$, if and only if $A u$ utters $x$ (or $S$ ) intending that, once $x$ (or $S$ ) is read for the first time: i) $x$ (or $S$ ) persistently should (be disposed to) cause propositional imagining that $p$ in her intended readership $R$, for as long as she is thinking of $F$ et all; ii) $R$ should recognize this intention; and iii) $R$ 's recognition of this intention should function as part of $R$ 's reason to propositionally imagine that $p$. (Stock 2017, S. 66; Hervorhebung im Original)

Demnach sind allein jene propositionalen Vorstellungen, die der Autor nicht bloß vorübergehend, sondern andauernd verschreibt, fiktionale Wahrheiten. Wenn Vorstellungen zuerst verschrieben, dann aber wieder zurückgezogen werden, sind es fiktionale Falschheiten. Es schien bloß eine Weile so, als seien es fiktionale Wahrheiten - weil mimetisch unzuverlässig erzählt wurde.

Ich sehe aber folgendes Problem an dieser Standardposition: Wenn den Propositionen der White-Fiktion derselbe fiktionale Wahrheitswert zugeteilt würde, 
wie allen anderen in Stiller fiktional falschen Propositionen - dann wären die positiv durch den Autor generierten, vorzustellenden und für die Interpretation des Werks relevanten Propositionen der White-Fiktion ein Teil derselben Menge an Propositionen, der auch alle Propositionen angehören, die nicht durch den Autor generiert wurden, nicht vorzustellen sind und für die Interpretation des Werks auch nicht relevant sind. Dort gehören sie aber nicht hin, weil wir mit einem Text wie Stiller offensichtlich nicht so umgehen. Wir behandeln die Propositionen der White-Fiktion beim Interpretieren nicht wie übliche fiktionale Falschheiten. Wir behandeln sie aber auch nicht wie übliche fiktionale Wahrheiten.

Die Proposition $w$ - dass es sich beim Protagonisten um White handelt -, ist nicht in demselben Sinne fiktional falsch wie zum Beispiel die Proposition, dass es sich beim Protagonisten um einen Analphabeten handelt (er verfasst, unabhängig davon, ob er White oder Stiller ist, ein Tagebuch). Die Proposition $w$ ist aber auch nicht in demselben Sinne fiktional wahr wie zum Beispiel die Proposition, dass es sich beim Protagonisten um einen Menschen handelt (er hat, wiederum unabhängig davon, ob er White oder Stiller ist, nur menschliche Eigenschaften).

\section{Aufspaltung einer fiktiven Welt}

Deshalb schlage ich vor, an diesem Punkt den Begriff der Autorisierung zu verwenden. Anders als Köppe und Kindt, die damit letztlich fiktionale Wahrheiten und fiktionale Falschheiten unterscheiden, sollen damit aber zwei Typen von fiktionalen Wahrheiten unterschieden werden.

Der Roman Stiller enthält demnach zwei fiktive Welten. Erstens die autorisierte fiktive Welt der Stiller-Fiktion, die aus einer ersten Menge an zu verknüpfenden fiktionalen Wahrheiten besteht. Zweitens die unautorisierte fiktive Welt der WhiteFiktion, die aus einer zweiten Menge an zu verknüpfenden fiktionalen Wahrheiten besteht.

Manche fiktionalen Wahrheiten teilen sich diese beiden fiktiven Welten, so zum Beispiel die Proposition, dass der Protagonist im Gefängnis in Zürich sitzt und sieben Hefte vollschreibt. Andere Propositionen sind nur in einer der beiden fiktiven Welten fiktional wahr, so zum Beispiel die Propositionen, dass es sich um Stiller bzw. um White handelt, der diese Hefte vollschreibt.

Nach dieser Sichtweise sind zwei durch den Autor generierte, aber sich widersprechende fiktionale Wahrheiten $p$ und non- $p$ (hier: $s$ und $w$ ) in verschiedenen fiktiven Welten der Fall. Dieses Sowohl-als-auch scheint mir erstens die Struktur von mimetisch unzuverlässig erzählten Erzählungen und zweitens unseren interpretativen Umgang mit solchen adäquater zu erfassen als das Entweder-oder der Standardposition. Nach der Standardposition kann in der einen und einzigen fiktiven Welt eines fiktionalen Werks letztlich nur $p$ oder non- $p$ der Fall sein wobei nach dieser Sicht höchstens unentscheidbar bleiben kann, was der Fall ist. 
Mimetisch unzuverlässiges Erzählen führt insofern notwendigerweise, so die These, zu einer Aufspaltung von einer fiktiven Welt in mindestens zwei fiktive Welten. In Stiller geschieht diese Aufspaltung mit dem ersten Satz der Gefängnisaufzeichnungen und der Generierung von zwei sich widersprechenden fiktionalen Wahrheiten. Das Resultat ist, wiederum mit einem Ausdruck von Kathleen Stock, eine multiple Fiktion (vgl. Stock 2017, S. 150, 169-174): Ein fiktionales Werk mit mehreren fiktiven Welten - also mindestens zwei distinkten Mengen an in der Vorstellung zu verknüpfenden fiktionalen Wahrheiten.

\section{Autorisierung fiktiver Welten}

Wenn nach dieser Aufspaltung im Rahmen einer Interpretation entschieden bzw. mit guten Gründen behauptet werden kann, dass eine fiktive Welt die autorisierte ist, handelt es sich, um an Matthias Aumüllers Terminologie anzuknüpfen (vgl. Aumüller 2018), um unzuverlässiges Erzählen mit geschlossener Funktion: Zuletzt ist eine fiktive Welt autorisiert, die andere(n) sind unautorisiert. Wenn aufgrund einer Interpretation nicht entschieden werden kann, dass eine fiktive Welt die autorisierte ist, handelt es sich um unzuverlässiges Erzählen mit offener Funktion: Zuletzt bleiben zwei oder mehr fiktive Welten neben einander stehen. ${ }^{13}$ Dabei handelt es sich um die Grundtypen am jeweiligen Ende einer Skala. Die Funktion des unzuverlässigen Erzählens ist also entweder (ganz) geschlossen oder mehr oder weniger offen.

In demselben Sinne verstehe ich Autorisierung als eine Frage des Grades. Im Fall von Stiller kann man zum Beispiel sagen, dass Frisch die Stiller-Fiktion stark autorisiert, aber gleichwohl nicht vollständig oder maximal oder eindeutig. Vor allem die Zahnarzt-Episode gegen Ende der Aufzeichnungen im siebten Heft gibt dem Leser nochmals einen Grund, um daran zu zweifeln, dass es sich beim Protagonisten um Stiller handelt. Ein Zahn, der laut Röntgenbild tot sein sollte, wenn es Stillers Zahn wäre, lebt noch:

[I]m Hinblick auf den alten Röntgen-Status, den sie in der Kartothek des Vorgängers gefunden haben, kann der junge Zahnarzt es einfach nicht fassen, daß mein Vierer-untenlinks noch lebt, meines Erachtens empfindlich genug, auch wenn es auf dem RöntgenStatus (man zeigt mir den Vierer-unten-links, wie ihn der verschollene Stiller hatte) ganz und gar nach einer toten Wurzel aussieht. „Merkwürdig“, murmelt er, „,merkwürdig.“ Dann klingelt er dem Fräulein. „Ist das wirklich der Röntgen-Status von Herrn Stiller?“ fragt er. „Sind Sie sicher?“” „Es steht doch drauf -“(Frisch 1976, S. 665)

\footnotetext{
${ }^{13}$ Weil in solchen Fällen nicht nur nicht entschiedenen werden kann, sondern erst gar nicht entschieden werden soll, welche fiktive Welt die autorisierte ist, scheint mir die Rede von unentscheidbarem unzuverlässigen Erzählen (vgl. Martinez und Scheffel 1999, S. 103; Köppe und Kindt 2014, S. 241) im Gegensatz zu unzuverlässigem Erzählen mit offener Funktion unpassend.
} 
Frisch hält so die White-Fiktion als Möglichkeit offen. ${ }^{14}$ Der Plot der StillerFiktion und der Plot der White-Fiktion verlaufen bis zuletzt neben einander. Die Konzeption des Romans erlaubt es, dass der Leser jeden Satz aus mindestens zwei Perspektiven interpretieren kann: aus der stark autorisierten Stiller-Perspektive einerseits, aus der schwach autorisierten White-Perspektive andererseits.

Was also ist unter Autorisierung zu verstehen? Sicher handelt es sich um eine Tätigkeit des Autors. Wenn er eine fiktive Welt autorisiert, gibt er dieser in irgendeiner Weise den Vorzug. Damit wird also vielleicht so etwas gesagt wie: Eine fiktive Welt ist die primäre oder realisierte oder aktuale - eine andere fiktive Welt hingegen die sekundäre oder potenzielle oder mögliche. Autorisierung auf diese Weise durch womöglich viel komplexere Begriffe zu erläutern, halte ich aber für kontraproduktiv. Eine explizite Bestimmung scheint mir zudem an dieser Stelle nicht nötig. Der Duden nennt als Synonyme unter anderem Befugnis, Berechtigung oder Erlaubnis (vgl. Dudenredaktion o.J.). In diesem alltagssprachlich üblichen Sinne ist Autorisierung auch hier zu verstehen: Der Autor gibt dem Leser die Befugnis, Berechtigung oder Erlaubnis, eine fiktive Welt einer anderen vorzuziehen. ${ }^{15}$

\section{Sein und Tun}

Auf diese Weise stellt Frisch in Stiller eine (nicht vollständig) autorisierte fiktive Welt einer (nicht vollständig) unautorisierten fiktiven Welt gegenüber. Für ein Verständnis des Romans müssen offensichtlich beide berücksichtigt werden. Mit der Feststellung, dass die Stiller-Fiktion stärker autorisiert ist als die White-Fiktion, hat eine Interpretation, die nach der Werkbedeutung fragt, nur begonnen. Mit dem ausgeführten Vorschlag kann, im besten Fall, erklärt werden, in welchem Sinne die White-Fiktion ein Teil des Romans ist und bleibt. ${ }^{16}$ Der Vorschlag soll dabei für alle Erzählungen gelten können, in denen sich der Autor der Technik des mimetisch unzuverlässigen Erzählens bedient: Einmal verschriebene Vorstellungen sind und bleiben fiktionale Wahrheiten, unterscheiden kann sich einzig ihr Autorisierungsgrad. Auf dieser Basis möchte ich mit zwei Inter-

\footnotetext{
${ }^{14}$ Vgl. zu dieser Sicht auch Weidermann (2010, S. 189-190).

${ }^{15} \mathrm{Zu}$ Waltons (ebenfalls nicht explizit bestimmter) Verwendung des Ausdrucks vgl. Walton (1990, S. 51). Vgl. auch Köppe und Kindt (2011, S. 83).

${ }^{16}$ Eine solche Erklärung können Vertreter der Standardposition - die also die Propositionen der White-Fiktion nicht als unautorisierte fiktionale Wahrheiten, sondern als fiktionale Falschheiten klassifizieren würden - meines Erachtens nicht liefern. Auch wenn Vertreter der Standardposition immer noch zwischen zwei Typen von fiktionalen Falschheiten - interpretationsrelevanten und nicht-interpretationsrelevanten - unterscheiden könnten, bleibt unklar, inwiefern die Propositionen der White-Fiktion Teil des Werks bleiben können, wenn fiktive Welten als Mengen von fiktionalen Wahrheiten und fiktionale Wahrheiten als Ergebnis von verschriebenen Vorstellungen verstanden werden.
} 
pretationsskizzen abschließen: Zunächst aus der Perspektive der Stiller-Fiktion, dann aus der Perspektive der White-Fiktion.

„Ich bin nicht Stiller!“ (Frisch 1976, S. 361) - Was sollen wir uns hier im Detail vorstellen? Nicht bei der ersten Lektüre des Satzes, sondern nach einer Interpretation des Romans? Wir sollen uns sicherlich nicht vorstellen, dass Stiller einfach nur lügt, wenn er sagt, er sei nicht Stiller. Er meint damit etwas anderes als bloß juristische Identität. Er glaubt oder hofft, nicht mehr der Stiller zu sein, der er gewesen ist, sondern sein neues, besseres Ich entworfen zu haben und nun nicht mehr so heißen zu können, wie sein altes, schlechtes Ich.

Eigentlich gibt er zu verstehen: „Ich will nicht mehr der Stiller sein, der ich gewesen bin!“ Die Art, wie er seine „Ich-bin-nicht-Stiller“-Behauptung in verschiedenen Variationen wiederholt, verdeutlicht dies. Immer wieder spricht er von diesem, ihrem Stiller, mit dem er nicht mehr identisch sein will, weil dieser, ihr Stiller in seiner eigenen Einschätzung ein Versager ist. Er hat versagt im Krieg und versagt in der Liebe. Was für ihn bedeutet: Er hat versagt als Mann. „Stiller scheint wirklich der Inbegriff einer männlichen Mimose gewesen zu sein“ (Frisch 1976, S. 457), urteilt Stiller als White über Stiller.

Dieser Stiller, der im Krieg nicht schießen konnte, dessen ,ganze jämmerliche Ehe“ (Frisch 1976, S. 496) mit Julika ein seelenzermürbender Krieg war, will er nach einem Selbstmordversuch nicht mehr sein. Statt Verantwortung zu übernehmen für sein Scheitern (ob nun real oder eingebildet oder ein wenig von beidem), statt Schwächen zu beheben und Fehler gutzumachen, will er sich als White neu erfinden: als einen in seiner Vorstellung starken Mann, ohne Neurosen, voller Potenz. ${ }^{17}$ Das ist aber genau jenes feige Verhalten, das Stiller als den Stiller entlarvt, der er nicht mehr sein will. ,[I]mmer kommst du mit deinen Hirngespinsten!““ (Frisch 1976, S. 408). So Julikas Urteil zu Stillers fixer Idee.

Am Ende scheint mir denn auch keine Einsicht oder gar Selbstannahme $\mathrm{zu}$ stehen - auch wenn der Staatsanwalt ${ }^{18}$ sowie manche Interpreten ${ }^{19}$ das so

\footnotetext{
${ }^{17}$ Vgl. zu Stillers Vorstellungen von Männlichkeit auch Rohner (2015, S. 30-65).

${ }^{18}$ Der Staatsanwalt schreibt in seinem Nachwort: „Sein Verstummen, wenn man es einmal so nennen will, war ja in der Tat ein wesentlicher, vielleicht sogar der entscheidende Schritt zu seiner inneren Befreiung, die wir nicht allein an unserem Freund zu erkennen vermochten, sondern deutlicher noch an seinen Nächsten, an einer kaum merklichen und eigentlich langsamen, jedoch wirklichen Verwandlung unseres Verhältnisses zu ihm. Es wurde möglich, sein Freund zu sein; Stiller war frei geworden von der Sucht, überzeugen zu wollen“ (Frisch 1976, S. 730).

${ }^{19}$ So wollen zum Beispiel Manger (1966), Harris (1968), Frühwald (1978) und Petersen (2002) eine jeweils mehr oder weniger vollständige Selbstannahme erkennen. Nach Harris ist Stiller „,zur Wahrhaftigkeit und zur endgültigen Erkenntnis gelangt, daß er zwar sein Ich zurückerobert, indem er sich selbst angenommen, seinem Du aber - also Julika - nicht dieselben Rechte gegönnt habe“ (1968, S. 695). Frühwald spricht von einer „radikalen Ich-Annahme“ (1978, S. 266). Petersen wiederum davon, dass es Stiller am Schluss schaffe, „ganz zu sich selbst zurückzufinden“ (2002, S. 116). Für Forschungsüberblicke und Zusammenfassungen der gängigen Interpretationen vgl. Schößler und Schwab (2004, S. 10-12) sowie Albarella (2003, S. 16-21).
} 
sehen -, sondern Resignation. Stiller ist einfach wieder derselbe. Er hat als Stiller, der White zu sein versucht hat, keine Entwicklung gemacht, die ihn von einem Punkt an einen anderen geführt hätte, sondern sich im Kreis gedreht. Stiller war bereits davon überzeugt, Julika seelisch ermordet zu haben. Trotzdem geht er - wissend, dass es sich um einen Fehler handelt - nach seiner White-Episode zu ihr zurück:

Warum ich zurückgekommen bin?! Das hast du nicht erlebt. Eine Idiotie, nichts anderes, eine Starrköpfigkeit! Begreifst du's denn nicht? Wenn du ein halbes Leben lang vor einer Tür gestanden und geklopft hast, Herrgott nochmal, erfolglos wie ich vor dieser Frau, vollkommen erfolglos, Herrgott nochmal - und dann geh du weiter! Vergiß sie, so eine Tür, die dich zehn Jahre versäumt hat! Gib's auf, geh weiter!... Was heißt da schon Liebe? Ich habe sie nicht vergessen können. Das ist alles. Wie man eine Niederlage nicht vergessen kann. Warum ich zurückgegangen bin? Aus Besoffenheit, mein Lieber, aus Trotz. Du mit deinen noblen Meinungen! Geh in ein Kasino, schau sie dir an, wie sie weiterspielen, wenn sie verlieren, immer weitersetzen. Genau so! Weil's einen Punkt gibt, wo sich das Aufgeben nicht mehr lohnt. Aus Trotz, ja, aus Eifersucht! Du kannst eine Frau verlieren, wenn du sie gewonnen hast. Soll einer kommen! Aber wenn du selber nie gewonnen hast, nie gefunden, nie erfüllt? Vergiß sie, so eine Türe, und laß andere eintreten, geh weiter! Recht hast du: Warum haben wir uns nicht getrennt? Weil ich feige bin. (Frisch 1976, S. 768)

Wenn es dann zuletzt, auf Büchners Lenz-Abschluss anspielend, heißt: „Stiller blieb in Glion und lebte allein“ (Frisch 1976, S. 780), ist das Ende auf ganzer Linie tragisch. Eine Selbstfindung ist ausgeblieben, Julika ist tot, und Stiller wird sich selbst nun auch als ihr physischer Mörder verurteilen. Stillers Scheitern ist also vollkommen. ${ }^{20}$ Wer bin ich - und was kann ich dagegen tun? Dies scheint mir die zentrale existenzphilosophische Frage, die Frisch derart verhandelt. Stiller handelt insofern durchaus auch von Identität als solcher - vor allem aber vom Leiden des Individuums an der eigenen Identität.

Der Roman stellt den Leser vor die Frage: Was wäre zu tun, wenn man wie Stiller nicht mag, wer man gewesen ist? Keine Option ist, das soll gezeigt werden, die eigenen Verfehlungen zu leugnen. Erstens toleriert die Gesellschaft diese Art von moralischer Hochstapelei nicht. Zweitens verliert ein Individuum ohne Vergangenheit erst recht seine Identität. Ein Leiden am Selbst kann nur durch anderes, besseres, moralischeres Handeln gemindert werden, was auch immer das im Detail heißen mag.

Stiller hingegen trägt weder Schuld noch Verantwortung. Er will alle Belastungen zum Verschwinden bringen, sie auslöschen, indem er in eine Phantasie flüchtet. Dies, obwohl er auch in seiner White-Rolle eigentlich weiß,

\footnotetext{
${ }^{20}$ Stiller wird, so Roman Bucheli, ,am Ende mit der Höchststrafe belegt“ (2013, S. 14). Ebenso urteilt Jurgensen über den am Ende allein zurückbleibenden Stiller: „Seine epische Wiedergabe durch Rolf unterstreicht den Verlust einer Vitalität, die sein eigentliches Wesen kennzeichnete. Resignation wird hier fälschlicherweise als Lösung empfunden“ (1972, S. 79). Und auch Hans Mayer erkannte kein gutes Ende: „Kein Weg nach Innen, keine Befreiung durch ein einfaches Leben, keine Katharsis durch die Kunst. Alles bleibt offen, ungelöst, unbewältigt“ (1963, S. 50).
} 
dass das nicht funktionieren kann: „Heute wieder sehr klar: das Versagen in unserem Leben läßt sich nicht begraben, und solange ich's versuche, komme ich aus dem Versagen nicht heraus, es gibt keine Flucht“ (Frisch 1976, S. 589).

Der Roman fordert aber auch eine Interpretation aus der Sicht der WhiteFiktion. In dieser fiktiven Welt sagt White die Wahrheit, wenn er behauptet, nicht Stiller zu sein. Der Staatsanwalt hingegen täuscht sich. ${ }^{21}$ Jedoch lernt White durch die Erzählungen von Julika, Sibylle und Rolf im Laufe seiner Haft diesen, ihren verschollenen Stiller derart gut kennen, dass er glaubt, dessen Identität annehmen zu können. Er fügt die verschiedenen Stiller-Bildnisse zu einer Stiller-Identität zusammen. Er gibt den Kampf um seine eigene Identität auf und nimmt die angebotene „Flucht in eine Rolle“ (Frisch 1976, S. 401) an. Er fügt sich - jedoch ohne jemals explizit zuzugeben, dass er Stiller sei - dem Umfeld, das darauf beharrt, dass er Stiller zu sein hat. Bereits nach dem Zahnarztbesuch war White durch den Kopf gegangen:

Vielleicht, ich frage mich, müßte man sich überall wehren, wo man verwechselt wird, und ich dürfte es keinem Empfangsfräulein durchlassen, daß sie mich als Herr Stiller verbucht; eine Sisyphos-Arbeit! (Frisch 1976, S. 666)

Diese Arbeit legt er endgültig nieder, als ihn die Justiz dazu verurteilt, Stiller zu sein. „Stiller war frei geworden von der Sucht, überzeugen zu wollen“ (Frisch 1976, S. 730), schreibt Rolf in seinem Nachwort dazu, wobei er Whites Resignation mit Stillers Freiheit verwechselt.

In einer fremden Rolle kann auch White nur scheitern. Selbst wenn Identität, wie der Roman weiterhin nahelegt, zumindest zum Teil durch eine Erzählung durch eine Auswahl, Ordnung und Verknüpfung von Ereignissen - konstruiert wird: Es muss sich dann doch um die eigene Erzählung handeln. Als Stiller wiederholt White bloß Stillers Fehler.

Stiller kann seine Identität nicht ablegen und White sein. White kann seine Identität nicht ablegen und Stiller sein. In ihrer Macht liegt einzig, bessere oder schlechtere Versionen ihrer selbst zu sein. An diesem Punkt finden die beiden Interpretationen ihren gemeinsamen Kern.

\section{Schluss}

Am Beispiel des Romans Stiller von Max Frisch habe ich versucht die These plausibel zu machen, dass mimetisch unzuverlässiges Erzählen zu einer Aufspaltung von einer fiktiven Welt in mehrere - potenziell unterschiedlich stark autorisierte - fiktive Welten führt. Dies scheint zumindest die Konsequenz aus der

\footnotetext{
${ }^{21}$ Und wie gesehen gibt es gute Gründe für dir Annahme, dass Rolf ein unzuverlässiger Erzähler ist: Besonders seine wenig überzeugende Erklärung von Stillers Entwicklung macht ihn verdächtig.
} 
etablierten fiktionstheoretischen Position zu sein, dass fiktionale Wahrheiten durch Vorstellungsaufforderungen generiert werden. Zusammengefasst:

(1) Fiktionale Wahrheiten sind vorzustellende Propositionen

(2) Fiktive Welten sind Mengen an zu verknüpfenden fiktionalen Wahrheiten

(3) Mimetisch unzuverlässiges Erzählen führt zu sich widersprechenden fiktionalen Wahrheiten

(4) Sich widersprechende fiktionale Wahrheiten führen zur Aufspaltung von einer fiktiven Welt in mindestens zwei fiktive Welten

(5.1) Bei mimetisch unzuverlässigem Erzählen mit (vollständig) geschlossener Funktion wird eine fiktive Welt autorisiert

(5.2) Bei mimetisch unzuverlässigem Erzählen mit (mehr oder weniger) offener Funktion stehen sich mindestens zwei fiktive Welten ohne vollständige Autorisierung gegenüber

\section{Literatur}

Albarella, Paola: Roman des Übergangs. Max Frischs „Stiller“ und die Romankunst um die Jahrhundertwende. Würzburg: Königshausen und Neumann, 2003.

Aumüller, Matthias: Offenheit und Geschlossenheit als Funktionen des unzuverlässigen Erzählens. Mit Interpretationsbeispielen anhand Texten von Ernst Weiß, Paul Zech und Stefan Zweig. In: Journal of Literary Theory 12.1 (2018), 127-150.

„Autorisierung“. Duden online. URL: https://www.duden.de/rechtschreibung/Autorisierung (18.11.2018).

Bucheli, Roman: Die Verbesserung des Ich. Max Frischs biografische und ästhetische Metamorphosen. In: Text + Kritik: Max Frisch. Hg. Hermann Korte. München: Edition Text + Kritik ${ }^{4} 2013,3-21$.

Frisch, Max: Stiller. Roman. In: Max Frisch. Gesammelte Werke in zeitlicher Folge, Bd. 3 (19491956). Hg. Hans Mayer unter Mitw. Walter Schmitz. Frankfurt am Main: Suhrkamp, 1976 [1954], 359-780.

Frühwald, Wolfgang: Parodie der Tradition. Das Problem der Originalität in Max Frischs Roman „Stiller“. In: Materialien zu Max Frisch ,Stiller‘. Hg. Walter Schmitz. Frankfurt am Main: Suhrkamp 1978, 256-268.

Gabriel, Gottfried: Erkenntnis. Berlin/Boston: De Gruyter, 2015.

Grice, Paul H.: Meaning. In: The Philosophical Review 66.3 (1957), 377-388.

Grice, Paul H.: Logic and Conversation. In: Peter Cole/Jerry Morgan (Hg.), Syntax and Semantics, Vol. 3. Speech Acts. New York 1975, 41-58.

Harris, Kathleen: Stiller (Max Frisch): Ich oder Nicht-Ich?, In: The German Quarterly 41:4 (1968), 689-697.

Jacke, Janina: Unreliabilty and Narrator Types. On the Application Area of ,Unreliable Narration'. In: Journal of Literary Theory 12.1 (2018), 3-28.

Jurgensen, Manfred: Max Frisch. Die Romane [1972]. Bern: Francke, ${ }^{2} 1976$.

Kindt, Tom: Unzuverlässiges Erzählen und literarische Moderne. Eine Untersuchung der Romane von Ernst Weiß. Tübingen: Max Niemeyer, 2008.

Köppe, Tilmann: Literatur und Erkenntnis. Studien zur kognitiven Signifikanz, fiktionaler literarischer Werke. Paderborn: Mentis, 2008.

Köppe, Tilmann und Tom Kindt: Unreliable Narration With a Narrator and Without. In: Journal of Literary Theory 5:1 (2011), 81-94.

Köppe, Tilmann und Tom Kindt: Erzähltheorie. Eine Einführung, Stuttgart: Reclam, 2014. 
Köppe, Tilmann: Fiktive Tatsachen. In: Tobias Klauk, Tilmann Köppe (Hg.), Fiktionalität. Ein interdisziplinäres Handbuch, Berlin/Boston: De Gruyter, 2014, 190-208.

Lang, Simone Elisabeth: „Zumindest hätte er sich all dies gewünscht.“ Unzuverlässiges Erzählen in der Heterodiegese. [im Erscheinen]

Lindblom, Victor: Ist es wirklich so schlimm? Zur Fiktionalität und Erzählkonzeption von Lukas Bärfuss' „Koala“. In: Text + Kritik: Lukas Bärfuss. Hg. Tom Kindt und Victor Lindblom. München: Edition Text + Kritik. 2020, 39-45.

Martinez, Matias und Scheffel Michael: Einführung in die Erzähltheorie, München: C.H. Beck, 1999.

Mayer, Hans: Dürrenmatt und Frisch. Anmerkungen, Pfullingen: Neske, 1963.

Petersen, Jürgen H.: Max Frisch, Stuttgart: Metzler, ${ }^{3} 2002$.

Rohner, Melanie: Farbbekenntnisse: Postkoloniale Perspektiven auf Max Frischs „Stiller“ und „Homo Faber", Bielefeld: Aisthesis, 2015.

Schößler, Franziska und Eva Schwab: Max Frisch, Stiller. Ein Roman. München: Oldenburg, 2004.

Stock, Kathleen: Only Imagine. Fiction, Interpretation, and Imagination. Oxford: Oxford University Press, 2017.

Stühring, Jan: Unreliability, Deception, and Fictional Facts. In: Journal of Literary Theory 5:1 (2011), 95-108.

Walton, Kendall: Mimesis as Make-Believe. On the Foundations of the Representational Arts.Cambridge, London: Harvard University Press, 1990.

Weidermann, Volker: Max Frisch. Sein Leben, seine Bücher. Köln: Kiepenheuer \& Witsch, 2010.

Wittgenstein, Ludwig: Tractatus logico-philosophicus. Frankfurt am Main: Suhrkamp 2006.

Zipfel, Frank: Fiktion, Fiktivität, Fiktionalität. Analysen zur Fiktion in der Literatur und zum Fiktionsbegriff in der Literaturwissenschaft. Berlin: Erich Schmidt, 2001.

Zipfel, Frank: Unreliable Narration and Fictional Truth. In: Journal of Literary Theory 5:1 (2011), 109-130.

Open Access Dieses Kapitel wird unter der Creative Commons Namensnennung 4.0 International Lizenz (http://creativecommons.org/licenses/by/4.0/deed.de) veröffentlicht, welche die Nutzung, Vervielfältigung, Bearbeitung, Verbreitung und Wiedergabe in jeglichem Medium und Format erlaubt, sofern Sie den/die ursprünglichen Autor(en) und die Quelle ordnungsgemäß nennen, einen Link zur Creative Commons Lizenz beifügen und angeben, ob Änderungen vorgenommen wurden.

Die in diesem Kapitel enthaltenen Bilder und sonstiges Drittmaterial unterliegen ebenfalls der genannten Creative Commons Lizenz, sofern sich aus der Abbildungslegende nichts anderes ergibt. Sofern das betreffende Material nicht unter der genannten Creative Commons Lizenz steht und die betreffende Handlung nicht nach gesetzlichen Vorschriften erlaubt ist, ist für die oben aufgeführten Weiterverwendungen des Materials die Einwilligung des jeweiligen Rechteinhabers einzuholen. 